\title{
Prevalencia de factores de riesgo cardiovascular en pilotos de aviación civil en Colombia en el año 2005
}

\author{
Cardiovascular risk factor prevalence in civil aviation pilots in \\ Colombia during 2005
}

Luis F. Arteaga-Arredondo y Hugo A. Fajardo-Rodríguez

Universidad Nacional de Colombia, luisfelipearteaga@yahoo.com.ar, hafajardor@unal.edu.co

Recibido 6 Junio 2009/Enviado para Modificación 17 Enero 2010/Aceptado 23 Abril 2010

\section{RESUMEN}

Objetivo La seguridad aérea depende de la interfase hombre-avión. La enfermedad coronaria es la causa más frecuente de incapacitación súbita en vuelo y como factores se encuentran los descritos como riesgo cardiovascular. Los pilotos probablemente tienen una prevalencia diferente a la población general.

Metodología Estudio descriptivo transversal. Se revisaron los registros médicos de los pilotos certificados por la Aeronáutica Civil de Colombia, entre enero y diciembre de 2005, seleccionándolos aleatoriamente hasta completar 614 historias clínicas, según muestras tomadas con un nivel de confianza del $95 \%$ y una precisión del $0.5 \%$. Se extrajeron los siguientes datos: Edad, presión arterial, tabaquismo, colesterol, glicemia, ejercicio, antecedentes, sexo, talla, horas de vuelo, tipo de licencia. Los datos fueron analizados mediante STATG 6 y los resultados se expresaron según la estadística descriptiva.

Resultados La prevalencia de factores de riesgo en pilotos fue: HTA 7,8 \%, Diabetes 1,3 \%, Hipercolesterolemia $36 \%$, Hipertrigliceridemia $36 \%$, HDL Bajo $36 \%$, LDL Alto $32 \%$, Tabaquismo $12.8 \%$, Obesidad $7 \%$ y Síndrome Metabólico en el $6 \%$. Hubo diferencia entre pilotos de primera y segunda clase, siendo los factores de riesgo más frecuentes en la segunda categoría.

Conclusiones La prevalencia de factores de riesgo difiere entre pilotos y la población general, se identifican como de alto riesgo según la escala de Framingham al $8 \%$ de pilotos, los cuales requieren de programas específicos y seguimiento estrecho para modificar el perfil de riesgo y mejorar la seguridad aérea.

Palabras Clave: Factores de riesgo cardiovascular, hipertensión, enfermedad coronaria (fuente: DeCS, BIREME).

\section{ABSTRACT}

Objective The human-flying machine interface relies heavily on flight safety. Coronary disease is the most commonly occurring sudden incapacitation during flight and involves factors described as leading to cardiovascular risk. Pilots probably have risk prevalence different to the rest of the population. 
Method This was a descriptive cross-sectional study. Medical histories from January to December 2005 for pilots certified by the Colombian Civil Aeronautic society were reviewed; these were randomly selected until 614 histories were obtained. A $95 \%$ confidence level and $0.5 \%$ accuracy level were used. Data was obtained regarding age, blood pressure, tobacco use, cholesterol, glycaemia, physical fitness, past history, gender, height, flying time and type of licence. STATG 6 software was used for analysing the data and the results were expressed as descriptive statistics.

Results Percentage risk factor prevalence in pilots was as follows: $7.8 \%$ suffered hypertension, $1.3 \%$ diabetes, $36 \%$ hypercholesterolemia, $36 \%$ hypertrigliceridaemia, $36 \%$ had low HDL, $32 \%$ high LDL, $12.8 \%$ tobacco use, $7 \%$ were obese and $6 \%$ suffered from metabolic syndrome. There were differences between pilots holding first and second class licences, more prevalent risks occurring amongst second class pilots.

Conclusion The prevalence of risk factors differs between pilots and the general population. According to the Framingham scale, $8 \%$ of the pilots were in the high risk group so they require specific programmes and strict follow-up for modifying the risk profile and improving flight safety.

Key Words: Cardiovascular risk factor, hypertension, coronary disease (source: $\mathrm{MeSH}, \mathrm{NLM}$

a seguridad de vuelo en el transporte aéreo depende del buen funcionamiento de la compleja "interfase" hombre - avión. Entre los posibles factores humanos del accidente aéreo se cuentan: errores de pericia, errores de decisión, errores de percepción, violaciones a la normatividad y la incapacidad súbita de los tripulantes en vuelo, los cuales han sido motivo de estudio en Medicina Aeroespacial. La enfermedad coronaria parece ser la causa más frecuente de incapacidad súbita total en vuelo (1). La pérdida de aptitud de vuelo por enfermedad coronaria con sus consecuencias socioeconómicas es un problema a resolver con medidas preventivas, diagnósticas, terapéuticas y rehabilitadoras. Para lograr este objetivo es necesario conocer la frecuencia y distribución de los factores de riesgo cardiovascular en dicha población. La población de pilotos es altamente seleccionada y probablemente la prevalencia de factores de riesgo cardiovascular sea diferente a la población general.

Al examinar las causas de descalificación médica y pérdida de licencia en pilotos profesionales, se encuentra que la cardiopatía isquémica es la primera causa de pérdida de licencia, por cuanto las enfermedades cardiovasculares están frecuentemente asociadas con el riesgo de incapacitación súbita o insidiosa y pueden intrínsecamente descalificar a una persona para la realización de funciones aeronáuticas (2). 
El factor humano es importante en un "80 \%" de los accidentes de aviación, pero las causas médicas solo cuentan en un "5\%" aproximadamente, y la mitad de éstas son de origen cardiovascular (7). Por su importancia decidimos realizar un primer estudio sobre la prevalencia de factores de riesgo cardiovascular en pilotos de aviación civil en nuestro país.

\section{METODOS}

El Universo estuvo constituido por todos los registros médicos ubicados en el archivo de historias clínicas de AEROCIVIL correspondientes a pilotos certificados, con los cuales se realizó un estudio de tipo descriptivo correlacional.

La muestra (11) se tomó a partir del universo de historias clínicas (4 374), entre enero y diciembre del año 2005 , teniendo en cuenta un nivel de confianza del $95 \%$, una precisión del $0.5 \%$ y una prevalencia estimada para el factor de riesgo mas frecuente del $45 \%$ (12), (obesidad) seleccionando 614 registros los cuales se revisaron escogiéndolos a través de números aleatorios.

Los criterios de inclusión fueron: Ser pilotos con licencia de primera clase certificados; (línea, comerciales de avión, comerciales de helicóptero e ingenieros de vuelo), pilotos de segunda clase y pilotos privados de avión y helicóptero (13). Los criterios de exclusión fueron: Pilotos de aviación militar y personal aeronáutico que no posea licencia que los habilita como pilotos PPL, PCA, PCH, PPA, PPH. Los IDV y los IV son titulares de licencia de piloto comercial (13).

Del examen de tipo anual aplicado a los solicitantes de certificado médico de primera clase (PTL y PCA-H) y segunda clase (PPA-H) se obtuvieron los datos correspondientes a los factores de riesgo cardiovasculares como: Edad, presión arterial, tabaquismo, colesterol total, colesterol HDL, glicemia, ejercicio, historia familiar de CHD prematura, obesidad. Se tomaron otros datos como sexo, peso, talla, IMC, triglicéridos, colesterol LDL, horas de vuelo, tipo de piloto según licencia.

Se tuvieron en cuenta las siguientes definiciones: Síndrome metabólico: Obesidad central (perímetro abdominal $\geq 90$ en hombres. Mujeres $\geq 80$ ) más dos de los siguientes 4 factores: Triglicéridos $\geq 150 \mathrm{mg} / \mathrm{dl}$, colesterol HDL bajo $<40$ $\mathrm{mg} / \mathrm{dl}$, hipertensión arterial previa o TA sistólica $>130$ y TA diastólica $\geq 85$ y glicemia alterada en ayunas $\geq 100 \mathrm{mg} / \mathrm{dl}$ o diabetes previamente diagnosticada. En este estudio se tomará IMC $\geq 30$ dado que no hay registro de perímetro abdominal en las historias clínicas. Riesgo cardiovascular: A 10 años (ATP III)' 
Bajo $<10 \%$. Medio: $\geq 10 \%$ y $\leq 20 \%$ y alto: $\geq 20 \%$. Primero se determinó el número de factores de riesgo y luego se aplicó la tabla de Framingham dando categorías 0,1 y 2 siendo de alto riesgo pacientes con enfermedad cardiovascular o equivalentes como diabetes y enfermedad ateroesclerótica, moderado riesgo a personas con múltiples factores de riesgo $(>2)$ y bajo a quienes tienen por lo menos un factor de riesgo o ninguno. Sobrepeso: IMC 25-29, obesidad >30, diabetes: glicemia $\geq 126 \mathrm{mg} / \mathrm{dl}$; colesterol total > 200mg \%, LDL >130mg \%, HDL $<40 \mathrm{mg} \%$.

La información obtenida del estudio se manejó con cautela y respetando el derecho a la privacidad. A las personas que obtuvieron resultados indicativos de riesgo cardiovascular significativo, fueron llamadas para continuar estudios en su respectiva EPS, para que establecieran el tratamiento apropiado según el caso.

Los datos se analizaron utilizando el software STATA 6 y se expresaron mediante proporción de prevalencias, medidas de tendencia central y variabilidad. Se realizaron tambien tablas de contingencia. Las diferencias entre promedios y porcentajes se examinaron mediante el uso de estadísticos no paramétricos (prueba de Mann Whitney y prueba de rangos con signo) y el uso de estadísticos para diferencia de proposisiones $\left(\mathrm{x}^{2}\right)$. En los casos donde fue posible, se realizó análisis de covariables.

\section{RESULTADOS}

Se revisaron 614 historias clínicas, 347 pilotos con licencia de primera clase y 267 con licencia de segunda clase, entre ellos 17 mujeres y 597 hombres, cuyas características generales se muestran en la Tabla 1.

Tabla 1. Características generales Pilotos de Aviación Civil

\begin{tabular}{lc}
\hline \multicolumn{1}{c}{ Variable } & Promedio \\
\hline Edad & 42.6 \\
Peso & 77.3 \\
Talla & 1.74 \\
Colesterol total & 186.8 \\
HDL & 44.9 \\
LDL & 111.9 \\
Triglicéridos & 159.8 \\
Glicemia & 89.5 \\
Horas de vuelo & 4046.1 \\
IMC & 25.3 \\
P sistólica & 120.9 \\
\hline
\end{tabular}


En la Tabla 2, se muestra la prevalencia de los principales factores de riesgo cardiovasculares encontrados:

Tabla 2. Resumen prevalencia de factores de riesgo cardiovascular

\begin{tabular}{lc}
\hline \multicolumn{1}{c}{ Factor De Riesgo } & Prevalencia (\%) \\
\hline Hipertensión arterial & 7.8 \\
Diabetes Mellitus & 1.3 \\
Hipercolesterolemia & 36.3 \\
Hipertrigliceridemia & 39.7 \\
HDL Bajo & 36.6 \\
Hipercolesterolemia LDL & 32.7 \\
Tabaquismo & 12.9 \\
Antecedente Familiar & 5.1 \\
Obesidad & 7 \\
Síndrome Metabólico & 6 \\
\hline
\end{tabular}

Hubo relación significativa entre presión sistólica y horas de vuelo $(\mathrm{p}=0.0312)$. En la clasificación del riesgo cardiovascular a los 10 años. Según Framingham se encontró de bajo riesgo $<10 \%$ al $79.8 \%$ de personal de vuelo, medio riesgo $10-20 \%$ al $12,2 \%$ y de alto riesgo, $>20 \%$ al $7,98 \%$. Al discriminar entre pilotos de primera y segunda clase se encuentran de bajo riesgo al $85,6 \%$ y $72.3 \%$; mediano riesgo al $10,7 \%$ y $14,2 \%$; y alto riesgo al $3.7 \%$ y $13,5 \%$ respectivamente.

Existe asociación entre las variables riesgo cardiovascular y tipo de licencia, se consideró "expuestos" a los pilotos privados o de segunda clase con un OR 4 (IC: $2,1+7,6$ ) ajustando por tabaquismo que es una variable de interacción. Es decir, existe una probabilidad cuatro veces mayor de tener un riesgo cardiovascular alto si posee licencia de segunda clase o si es piloto privado. Tal como se muestra en la Tabla 3.

Tabla 3. Prevalencia de Factores de Riesgo según clase de licencia en pilotos

\begin{tabular}{lccc}
\hline \multirow{2}{*}{ Factor de Riesgo } & \multicolumn{2}{c}{ Prevalencia \% } & \multirow{2}{*}{$\begin{array}{c}\text { 2 Pearson } \\
\text { Valor P }\end{array}$} \\
\cline { 2 - 3 } & \multicolumn{1}{c}{$\mathbf{1}^{\text {a }}$ Clase } & $\mathbf{2}^{\text {a }}$ Clase & 0.014 \\
\hline HTA & 5,5 & 10,8 & 0.275 \\
Diabetes & 0,9 & 1,9 & 0.880 \\
Hipercolesterolemia & 37,2 & 35,2 & 0.178 \\
Hipertrigliceridemia & 42 & 36,7 & 0.585 \\
HDL Bajo & 36 & 37,5 & 0.564 \\
LDL & 31,8 & 33 & 0.000 \\
Tabaquismo & 5,8 & 22,1 & 0.104 \\
Obesidad & 6,9 & 7,1 & 0.093 \\
Antecedente familiar & 3,8 & 6,7 & 0.513 \\
Síndrome metabólico & 5,5 & 6,7 & \\
\hline
\end{tabular}


Los factores de riesgo de mayor prevalencia fueron hipertrigiceridemia y HDL Bajo e hipercolesterolemia, el OR por condición clínica fue: HTA 2.1, IC $(1,16-3,8)$, Tabaquismo 4,63 (2,7-7,88), Riesgo cardiovascular conjunto 4,0 (2,09$7,63)$.

\section{DISCUSIÓN}

La prevalencia en la población general de los factores de riesgo cardiovasculares ha sido: De HTA23,7 \%, diabetes 5,2 \%, hipertrigliceridemia $24,1 \%$, tabaquismo $26,1 \%$ y obesidad $45,2 \%$. Diferencia apreciable con la prevalencia de factores de riesgo en pilotos aunque no significativa, exceptuando obesidad y tabaquismo $(\mathrm{p}=0.000)$. Sin embargo en pilotos es frecuente el sobrepeso.

La enfermedad coronaria es la causa principal de muerte y discapacidad en el mundo, en especial después de los cuarenta años, también afecta significativamente a pilotos con un $25 \%$ de los casos registrados. Es responsable de una tercera parte de la pérdida de licencia. La experiencia de la oficina aeromédica de la Asociación de Pilotos de Aerolínea (ACPA) es que el $23 \%$ tienen enfermedades cardiovasculares y constituyen el $35 \%$ de la discapacidad. Es causa frecuente de dispensas, luego es deseable identificar tempranamente a pilotos con riesgo cardiovascular para intervenirlos en forma enérgica y evitar la discapacidad, la dispensa y muerte de origen cardiovascular.

En resumen la prevalencia de factores de riesgo cardiovascular en pilotos de aviación civil en Colombia en el 2005 oscila entre 1,3\% para diabetes y 39,7 \% hipertrigliceridemia. El tabaquismo e HTA son de mayor prevalencia en pilotos privados y mediante el estudio se ha identificado $8 \%$ de pilotos con alto riesgo cardiovascular que ameritan una intervención prioritaria para modificar el perfil de riesgo y un control o seguimiento estrecho en programas de prevención efectivos.

Los efectos sobre el riesgo de incapacitación médica en vuelo pueden ser modificados. La regla del $1 \%$ fue basada en la incapacitación cardiovascular y muerte cardiovascular, según tasas anuales de mortalidad. Un piloto que tenga un riesgo de incapacitación súbita anual del $1 \%$ se considera satisfactorio para operar cabinas múltiples, pero podría no serlo para operar cabinas únicas.

El cálculo del riesgo cardiovascular es esencial para determinar la pronta efectividad del tratamiento preventivo y tiene un papel fundamental en la reducción de las tasas de mortalidad cardiovascular y en los efectos sobre la 
incapacitación medica en vuelo. El cálculo del riesgo se hizo con tablas de otras poblaciones y cabria alguna limitación de su aplicación no validada en nuestro país. Sin embargo, el riesgo Framinghan ha sido mundialmente aceptado

\section{REFERENCIAS}

1. Evans A, Rainford D. medical santadards for aircrew. En: Ernsting J (ed). Aviation Medicine third edition. Oxford Butterworth Heinemann 1999; 217-231.

2. OACI Manual de Medicina Aeronáutica Civil segunda ed. 1985; III-1: III-1-20.

3. Illingworth R. Management of hipercolesterolemia. The Medical Clinics of North America 2000; 84 23-38.

4. Fernández-García F. Manual de Médico de Vuelo. Ministerio de Defensa. Madrid España. 2000; 241-43.

5. Jones C. How's your ticker? Flight Safety Australia. January-February 2003; 24-31.

6. Sharma JK. Clinical and angiographic profile of aircrew with coronary heart disease undergoing cardiac evaluation before being reflighted. Ind. J. Aerospace Medicine 2002; 46(2); 32-38.

7. García-Cosio F. Guías de Práctica Clínica de la Sociedad Española de Cardiología sobre conducción de vehículos, pilotaje de aviones y actividades subacuáticas en cardiópatas. Rev. Española de Cardiología 2001;54:476-90.

8. Bailey D, Gilleran L. Waivefor disqualifying medical conditions in US naval aviation personnel. Aviation Space Environmetal. Med. 1995; 66: 401-07.

9. Executive Summary of the third report of the National Cholesterol Education Program (NCEP) Expert panel on detection, evaluation and treatment of high blood cholesterol in adults (ATP III). JAMA 2001; 285: 2486-97.

10. Mltchel S. Flight Safety and Medical Incapacitation Risk of Airline Pilots. Aviation Space Environ Med. 2004; 75:260-8.

11. Fajardo H, Gutiérrez A, Navarrete, Barrero J.. Prevalencia de factores de riesgo cardiovascular. Localidad de los Mártires. Bogotá Colombia. Revista Facultad de Medicina Universidad Nacional de Colombia. 2003; 51(4): 198-202.

12. Sánchez R. Los estudios Transversales. En: Ardila E. Estrategias de Investigación en Medicina Clínica. Bogotá Manual Moderno 2001; 84-85.

13. The IDF consensus wordlwide definition of the metabolic syndrome. Internacional Diabetes Federation, Bruselas, Belgium; 2005. 